\title{
An analysis of adverse events and human error associated with the imaging of patients at a major trauma centre in South Africa
}

\author{
A A Bashir, ${ }^{1} \mathrm{MB}$ ChB; V Y Kong, ${ }^{2,3} \mathrm{MB} \mathrm{ChB}, \mathrm{ChM}, \mathrm{MSc}, \mathrm{PhD}$, MRCS (Ed); J J P Buitendag, ${ }^{4} \mathrm{MB}$ ChB, MMedSci; V Manchev, ${ }^{3} \mathrm{FCS}(\mathrm{SA})$; \\ W Bekker, ${ }^{3}$ FCS (SA), Cert Trauma Surgery (SA); J L Bruce, ${ }^{3}$ MB ChB, FCS (SA), G L Laing, ${ }^{3}$ MB ChB, MSc, FCS (SA), \\ Cert Trauma Surgery (SA), PhD; P Brysiewicz, ${ }^{5}$ BSocSc, BA, MCur, PhD; D L Clarke, ${ }^{2,3}$ MPhil, MBA, PhD, FCS (SA) \\ ${ }^{1}$ Department of Radiology, Nelson R Mandela School of Medicine, College of Health Sciences, University of KwaZulu-Natal, Durban, South Africa \\ ${ }^{2}$ Department of Surgery, Faculty of Health Sciences, University of the Witwatersrand, Johannesburg, South Africa \\ ${ }^{3}$ Department of Surgery, Nelson R Mandela School of Medicine, College of Health Sciences, University of KwaZulu-Natal, Durban, South Africa \\ ${ }^{4}$ Department of Surgery, Tygerberg Hospital and Faculty of Medicine and Health Sciences, Stellenbosch University, Cape Town, South Africa \\ ${ }^{5}$ School of Nursing and Public Health, College of Health Sciences, University of KwaZulu-Natal, South Africa
}

Corresponding author: A A Bashir (docabashir@gmail.com)

Background. There is growing realisation that human error contributes significantly to morbidity and mortality in modern healthcare. A number of taxonomies and classification systems have been developed in an attempt to categorise errors and quantify their impact. Objectives. To record and identify adverse events and errors as they impacted on acute trauma patients undergoing a computed tomography (CT) scan, and then quantify the effect this had on the individual patients. It is hoped that these data will provide evidence to develop error prevention programmes designed to reduce the incidence of human error.

Methods. The trauma database was interrogated for the period December 2012 - April 2017. All patients aged $>18$ years who underwent a CT scan for blunt trauma were included. All recorded morbidity for these patients was reviewed.

Results. During the period under review, a total of 1566 patients required a CT scan at our institution following blunt trauma. Of these, 192 (12.3\%, 134 male and 58 female) experienced an error related to the process of undergoing a CT scan. Of 755 patients who underwent a CT scan with intravenous contrast, detailed results were available for 312, and of these 46 (14.7\%) had an acute deterioration in renal function. According to Chang's taxonomy, physical harm occurred as follows: grade I $n=6$, grade II $n=62$, grade III $n=45$, grade IV $n=11$, grade $\mathrm{V} n=27$, grade VI $n=21$, grade VII $n=15$, grade VIII $n=3$ and grade IX $n=2$. Adverse events were performing an unnecessary scan ( $n=24)$, omitting an indicated scan ( $n=23)$, performing the scan incorrectly $(n=8)$, scanning the wrong body part $(n=7)$, equipment failure $(n=18)$, omitting treatment following the scan $(n=6)$, incorrect interpretation of the scan $(n=65)$, deterioration during the scan $(n=6)$ and others $(n=35)$. The setting for the error was the ward $(n=19)$, the radiology suite $(n=126)$, the emergency department $(n=45)$ and the operating theatre $(n=2)$. The staff responsible for the adverse events were medical $(n=155)$, nursing $(n=4)$ and radiology staff $(n=15)$. There were 67 errors of commission and 125 errors of omission. The primary cause was a planning problem in 78 cases and an execution problem in 114.

Conclusions. Errors and adverse events related to obtaining a CT scan following blunt polytrauma are not uncommon and may impact significantly on the patient. Communication is essential to eliminate errors related to performing the wrong type of scan. The commonest errors relate to misinterpretation of the scan.

S Afr Med J 2019;109(9):693-697. https://doi.org/10.7196/SAMJ.2019.v109i9.13726

There is a growing realisation that human error contributes significantly to morbidity and mortality in modern healthcare. With the ever-increasing reliance on complex technology and the multidisciplinary team approach to modern healthcare, many opportunities exist for human error to impact on clinical outcome. ${ }^{[1-3]}$ In response, there has been a renewed focus on the study of human error and its potential effect in healthcare settings. ${ }^{[4-6]}$ This interest was stimulated by the publication of the monograph $T o$ Err is Human ${ }^{[1]}$ in 2000. Since then, a number of taxonomies and classification systems have been developed in an attempt to categorise errors and quantify their impact. ${ }^{[2,3]}$ These taxonomies have been based on work by industrial psychologists such as James Reason, who began to study human error in diverse industries such as aviation, nuclear power and rail transportation. ${ }^{[2]}$ All these industries have managed to develop enviable safety records over the past half century as they applied the lessons of error theory. However, the same cannot be said for healthcare. Despite the call made in To Err is Human, it would appear that applying error prevention strategies to healthcare is more difficult than applying it to large organisations. This does not imply that the attempt should be abandoned, but rather that ongoing research is required to better define the spectrum and impact of error in health systems. ${ }^{[1-7]}$ This problem is especially relevant in developing countries such as South Africa (SA), where resources are much more limited than in developed countries yet the impact of error on patient outcome is just as important. However, there is currently very limited literature that focuses on the developing world setting.

\section{Objectives}

The present study was a direct response to this unmet need and focused on a single component of a complex therapeutic pathway, namely advanced imaging in the acute care of the polytrauma patient. It sought to record and identify adverse events and errors as they impacted on acute trauma patients undergoing a computed tomography (CT) scan and then to quantify the effect this had on 
the individual patients. It is hoped that this knowledge will provide evidence to develop error prevention programmes designed to reduce the incidence of human error and limit its impact on individual patients.

\section{Methods \\ Clinical setting}

The city of Pietermaritzburg is the capital of KwaZulu-Natal (KZN) Province, SA, and the largest city in the western half of KZN, with one million inhabitants. The city is the referral point for western $\mathrm{KZN}$, which is a predominantly rural area with a population of two million people. The Pietermaritzburg Metropolitan Trauma Service provides and supervises trauma care across the city and maintains a prospective digital trauma registry for the region. Ethics approval to maintain the registry was obtained from the Biomedical Research Ethics Committee of the University of KwaZulu-Natal (ref. no. BCA221/13). This digital registry, called the Hybrid Electronic Medical Registry, is unique and has been discussed in the literature. ${ }^{[7]}$ The clerking medical staff enter the data onto an electronic preprepared clerking sheet. This is the process for all new admissions, meaning that clinical data are entered in real time. As the data are entered, they are directly incorporated into the registry. The completed pre-prepared clerking sheet is then printed and becomes the patient's clinical record. A similar process is followed at operation and at discharge. This system combines the functions of a medical registry and a medical record system. It also combines an electronic system with a paper-based system. ${ }^{[7]}$

\section{Management algorithms}

All blunt trauma patients are managed according to standard protocols. Patients who do not respond undergo a FAST (focused assessment with sonography for trauma) scan and are then expedited to the operating theatre if there is free intra-abdominal fluid. All other patients are then selected for a CT scan based on the mechanism of injury and clinical findings.

\section{Taxonomy of error}

Chang's taxonomy was used in this study. This taxonomy was used by the Joint Commission on Accreditation of Healthcare Organizations to produce a standardised nomenclature for the taxonomy of adverse outcomes. It classifies error into five complementary root nodes, which equate to the general descriptive terms in parentheses below. ${ }^{[3]}$

- Impact (How bad was it?). The taxonomy attempts to grade the impact of each adverse event on the individual patient and consists of three subclassifications that discriminate between 18 types of outcomes or effects. The harm index characterises the degree of harm experienced, and this can range from no harm to temporary or permanent impairment of physical or psychological function. The taxonomy also distinguishes between the medical (psychological or physical) and non-medical (legal, social or economic) impact of the adverse event.

- Type (What went wrong?). This refers to the processes of care that failed. We divide the processes of care into broad categories, namely errors of resuscitation, errors of assessment, operative or technical error, and logistical failure. A patient may experience any number of combinations of failed processes.

- Domain (Where did it go wrong?). The physical location where the event took place.

- Cause (Why did it go wrong?). The causes are divided into errors of planning, errors of execution, errors of omission (failure to undertake a necessary action) and errors of commission (the performance of an inappropriate action). Resuscitation and logistical failures are errors of execution, while assessment failures are errors of planning.

- Prevention (What are we going to do about it?). All error reduction programmes need to develop interventions to reduce the incidence of error and to limit its effect.

\section{Grading of morbidity}

The Clavien-Dindo grading system ${ }^{[8]}$ for all surgical complications was introduced in 2004. It is reproduced in Table 1. This grading system has been widely adopted and is a useful way of characterising the impact of an adverse event on an individual patient.

\section{Interrogation of the trauma database}

The trauma database was interrogated for the period December 2012 - April 2017. All patients aged $>18$ years who underwent a CT scan for blunt trauma were included. All recorded morbidity for these patients was reviewed. Morbidities not related to the actual process of obtaining a CT scan were excluded, following a modified Delphi technique. Once consensus had been achieved on which morbidities were directly associated with the process of obtaining a CT scan, this cohort became the study group.

\section{Statistical analysis}

Means and standard deviations are reported for normally distributed data, and medians and interquartile ranges for data not normally distributed. All data analyses were performed using SPSS 24.0 for Mac (SPSS, USA).

\begin{tabular}{|c|c|}
\hline Grade I & $\begin{array}{l}\text { Any deviation from the normal postoperative course not requiring surgical, endoscopic or radiological intervention. } \\
\text { This includes the need for certain drugs (e.g. antiemetics, antipyretics, analgesics, diuretics and electrolytes), treatment with } \\
\text { physiotherapy, and wound infections that are opened at the bedside }\end{array}$ \\
\hline Grade II & $\begin{array}{l}\text { Complications requiring drug treatments other than those allowed for grade I complications; these include blood transfusion } \\
\text { and total parenteral nutrition }\end{array}$ \\
\hline Grade III & $\begin{array}{l}\text { Complications requiring surgical, endoscopic or radiological intervention } \\
\text { - Grade IIIa: intervention not under general anaesthetic } \\
\text { - Grade IIIb: intervention under general anaesthetic }\end{array}$ \\
\hline Grade IV & $\begin{array}{l}\text { Life-threatening complications, including central nervous system complications (e.g. brain haemorrhage, ischaemic stroke, } \\
\text { subarachnoid haemorrhage) that require intensive care, but excluding transient ischaemic attacks } \\
\text { - Grade IVa: single-organ dysfunction (including dialysis) } \\
\text { - Grade IVb: multi-organ dysfunction }\end{array}$ \\
\hline Grade V & Death \\
\hline
\end{tabular}




\section{Results}

During the period under review, 1566 patients required a CT scan at our institution following blunt trauma. Of 755 patients who underwent a CT scan with intravenous contrast, 192 (12.3\%) experienced an error or adverse event related to the process of undergoing a CT scan; 134 of these patients were male and 58 female. The racial breakdown was black African $n=165$, Asian $n=11$, white $n=10$ and coloured $n=6$. Table 2 summarises the demographic characteristics.

\section{Impact}

There were legal implications in 31 cases, social implications in 136 and economic implications in 137. Morbidity is documented and classified against Chang's impact score and the Clavien-Dindo grading system in Tables 3 and 4, respectively. Two patients in this cohort ultimately died. One was sent to CT scanning inappropriately instead of being expedited to the operating theatre, and the other, who had a massive head injury, died shortly after returning from the CT scan. Of the 755 patients who underwent a CT scan with intravenous contrast, detailed results were available for 312 , and of these $46(14.7 \%)$ experienced an acute deterioration in renal function.

\begin{tabular}{ll} 
Table 2. Demographics of the patient cohort $(N=192)$ \\
\hline Sex, $n(\%)$ & $134(69.8)$ \\
Male & $58(30.2)$ \\
Female & 37 \\
Age (years), median & \\
Race, $n(\%)$ & $165(85.9)$ \\
Black & $6(3.1)$ \\
Coloured & $10(5.2)$ \\
White & $11(5.7)$ \\
Asian &
\end{tabular}

Table 3. Impact of the adverse events, according to Chang's taxonomy ${ }^{[3]}(N=192)$

\begin{tabular}{ll}
\hline & $\boldsymbol{n}(\%)$ \\
\hline Physical degree of harm & $6(3.1)$ \\
I & $62(32.3)$ \\
II & $45(23.4)$ \\
III & $11(5.7)$ \\
IV & $27(14.1)$ \\
V & $21(10.9)$ \\
VI & $15(7.8)$ \\
VII & $3(1.6)$ \\
VIII & $2(1.0)$ \\
IX & \\
Psychological degree of harm & $57(29.7)$ \\
I & $74(38.5)$ \\
II & $40(20.8)$ \\
III & $7(3.6)$ \\
IV & $9(4.7)$ \\
V & $5(2.6)$ \\
VI & $31(16.1)$ \\
Legal implications & $136(70.8)$ \\
Social implications & $137(71.3)$ \\
Economic implications & \\
&
\end{tabular}

\section{Type}

The adverse events included performing an unnecessary scan $(n=24)$, omitting an indicated scan $(n=23)$, performing the scan incorrectly ( $n=8)$, scanning the wrong body part $(n=7)$, equipment failure $(n=18)$, omitting treatment following the scan $(n=6)$, incorrect interpretation of the scan $(n=65)$, deterioration during or shortly after the scan ( $n=18$ cases, of which 6 occurred during the CT scan and required an intervention), and miscellaneous ( $n=35)$. In 21 cases a significant injury was missed. Deterioration during the CT scan occurred for a number of reasons. For example, a patient was sent for a CT scan inappropriately; his condition had not stabilised, and he should have been expedited to the operating theatre. Another patient had an undiagnosed tension pneumothorax, and 2 had dislodged endotracheal tubes. Two patients had non-salvageable head injuries and deteriorated during transfer back from the CT scanner. All 6 of these patients required an intervention while in the CT scan room to address their deterioration. Misinterpretation of CT scan images resulted in missed or delayed diagnosis of a variety of injuries, some of which were potentially serious and included missed vascular injuries $(n=7)$, missed intra-abdominal injuries $(n=6)$ and missed spinal injuries $(n=9)$. In 2 cases, reports were captured under the wrong patient name and this created clinical confusion. Table 5 summarises the types of errors.

\section{Domain}

The setting for the error was the ward $(n=19)$, the radiology suite $(n=126)$, the emergency department $(n=45)$ and the operating theatre $(n=2)$. The staff responsible for the adverse event were medical ( $n=155)$, nursing $(n=4)$ and radiology staff $(n=15)$. Table 6 summarises the domains where the adverse events occurred.

\section{Cause}

There were 67 errors of commission and 125 errors of omission. The primary cause was a planning problem in 78 cases and an execution problem in 114. There was no evidence of intentional error, recklessness or willful negligence in this cohort. There were 28 systems-related errors, and the rest were human errors. Table 7 summarises the causes of the errors.

\begin{tabular}{ll}
\multicolumn{2}{l}{ Table 4. Clavien-Dindo grades $^{[8]}(\boldsymbol{N}=\mathbf{1 9 2})$} \\
\hline I & $\boldsymbol{n}(\%)$ \\
II & $64(33.3)$ \\
IIIa & $77(40.1)$ \\
IIIb & $14(7.3)$ \\
IVa & $33(17.2)$ \\
V & $2(1.0)$ \\
& $2(1.0)$
\end{tabular}

Table 5. Types of adverse events $(N=192)$

\begin{tabular}{ll}
\hline & $\boldsymbol{n}(\%)$ \\
\hline Performing an unnecessary scan & $24(12.5)$ \\
Omitting an indicated scan & $23(11.0)$ \\
Performing the scan incorrectly & $8(4.2)$ \\
Scanning the wrong body part & $7(3.6)$ \\
Equipment failure & $18(9.4)$ \\
Omitting treatment following the scan & $6(3.1)$ \\
Incorrect interpretation of scan & $65(33.9)$ \\
Deterioration in the scan room & $6(3.1)$ \\
Other & $35(18.2)$
\end{tabular}




\begin{tabular}{ll} 
Table 6. Domain $(\boldsymbol{N = 1 9 2})$ & \\
\hline & $\boldsymbol{n}(\%)$ \\
\hline Setting in which the event occurred & \\
$\quad$ Radiology suite & $126(65.6)$ \\
Emergency department & $45(23.4)$ \\
Ward & $19(9.9)$ \\
$\quad$ Operating theatre & $2(1.0)$ \\
Staff involved & \\
Doctor & $155(80.7)$ \\
Nursing staff & $4(2.1)$ \\
Radiology staff & $15(7.8)$ \\
System & $18(9.4)$
\end{tabular}

Table 7. Classification of the causes of adverse events $(N=192)$

\begin{tabular}{ll}
\hline & $\boldsymbol{n}(\%)$ \\
\hline Human error & $164(85.4)$ \\
System error & $28(14.6)$ \\
Negligence error & 0 \\
Recklessness error & 0 \\
Intentional rule violations error & 0 \\
Cause & \\
$\quad$ Primary cause & \\
$\quad$ Error in planning & $78(40.6)$ \\
$\quad$ Error in execution & $114(59.4)$ \\
$\quad$ Secondary cause & \\
$\quad$ Error of commission & $67(34.9)$ \\
$\quad$ Error of omission & $125(65.1)$
\end{tabular}

\section{Discussion}

Advanced imaging is one of the cornerstones of modern trauma care, and CT scans allow for accurate and reliable imaging of the entire patient, which has facilitated modern non-operative approaches to trauma care. However, the process of obtaining imaging, similar to any other process of care, is susceptible to error and adverse events. The contemporary interest in understanding and quantifying adverse events and human error is derived from the realisation that error and adverse events have a significant impact on morbidity and even mortality and are costly to both the individual patient and the healthcare system as a whole. ${ }^{[1,7]}$ The aviation and nuclear power industries have developed highly successful interventions to improve safety and reduce both the incidence and the impact of adverse events. The challenge is to apply these lessons to modern healthcare. Modern healthcare is also a complex dynamic system involving multiple role players who need to work as a team to deliver care, and it makes logical sense that the lessons from aviation can be applied to healthcare. ${ }^{[1,7]}$

The CT scan is a dangerous environment for a trauma patient, as it is usually remote from the resuscitation area and the patient is relatively unmonitored during the procedure. All efforts must be made to ensure that the patient is in a physiological condition to withstand transfer to the CT scan. Sending a patient who is acidotic and not responding to resuscitation is an example of cognitive lock-out and represents protocol violation. This is an active error, or error of commission. Similarly, not excluding a potential tension pneumothorax is an error of assessment. The incidence of deterioration during the CT scan in our series (6/1 566) was $0.4 \%$. This is a serious error, and efforts must be directed towards preventing it. The adage of the CT scanner being the 'donut of death' has its basis in this error. ${ }^{[1,7]}$
A common cause of error is poor communication, both between members of the trauma team and between members of the medical team and radiology. Poor communication resulted in omitting an indicated scan, performing the scan incorrectly, and scanning the wrong body part. These errors have a cumulative effect on the patient, as they delay diagnosis and/or exclusion of an injury and necessitate repeat scans.

The commonest errors related to CT scanning are errors of interpretation of the images. ${ }^{[9-11]}$ This can have serious consequences for patients. We had 65 erroneous reports out of a total of 1566 , which gives an error rate of $4 \%$. False-positive results can result in unnecessary operations, and false-negative scans can delay intervention. The commonest areas of misinterpretation include the spine, the vasculature and intra-abdominal pathology. The incorrect interpretation of CT images is a multifactorial problem that can best be understood as the result of dissonance directly impairing cognitive capacity and function. An error rate of $4 \%$ is well within the range of internationally reported rates. Several strategies need to be implemented to reduce this rate and to limit the impact of these reporting errors. ${ }^{[12,13]}$ Most of these approaches revolve around the use of practices where each scan is reviewed by both the on-duty radiologist and the requesting surgeon. Understanding the mechanism of injury is essential to interpret CT findings, and without clinical insight, radiologists may fail to appreciate the significance of certain findings. ${ }^{[9,10]}$ Newer approaches include structured reporting, voice-recognition dictation and computeraided detection and assessment of images.

The incidence of so-called contrast-induced nephropathy following a contrast-enhanced CT scan is controversial. Some experts do not consider that it is a distinct entity, while others see it as a real phenomenon. In our cohort of patients who underwent a contrastenhanced CT scan, there was a significant incidence of deterioration in renal function. In our environment all patients undergo aggressive fluid resuscitation, and a good urine output is established before sending patients for a CT scan. ${ }^{[14]}$

The response to these errors needs to be multifactorial, and this research should inform potential changes in practice. The major move in surgical safety has been adoption of the so-called surgical checklist prior to operation. ${ }^{[15]}$ This approach has been shown to reduce postoperative morbidity and mortality. Extending it to a preCT scan checklist for trauma patients may help to reduce these errors. It would force staff to reassess patients in a structured manner before sending them for a CT scan. The important issues to check are the response to resuscitation and the indication for the CT scan. Patients who are transient responders or have an indication for surgery must be clearly identified and not sent for a CT scan. The checklist would identify patients at risk of acute kidney injury and would help to reduce errors of communication and ensure that the correct scan is performed on the correct patient. In addition, a direct and accurate hand-over between requesting staff and radiology staff will ensure that the mechanism is correctly understood by the reporting staff, which may help them to identify injuries.

\section{Study limitations}

There are a number of limitations to this study. Its retrospective nature made detailed root-cause analysis of specific errors difficult, as the original protagonists were no longer readily accessible to report on the exact sequence of events.

\section{Conclusions}

Errors and adverse events related to obtaining a CT scan following blunt polytrauma are not uncommon and may impact significantly 
on the patient. They include physiological deterioration during the CT scan, breakdowns in communication resulting in inappropriate scans being performed, misinterpretation of the CT scan images, and contrast-induced nephropathy. Efforts must be directed towards preventing these errors and limiting their impact on patients.

Declaration. This publication was a requirement for AAB's MMedSci degree.

Acknowledgements. None.

Author contributions. AAB: primary author, data analysis; VYK: manuscript conceptualisation; JJPB: analysis and statistics, drafting; VM: data analysis; WB: drafting, reference checking; JLB: manuscript editing, analysis, database maintenance and data retrieval; GLL: design of data capture instrument; PB: drafting, reference checking; DLC: senior author, general supervision, co-ordination and assistance at all levels.

Funding. None.

Conflicts of interest. None.

1. Kohn LT, Corrigan JM, Donaldson MS, eds. To Err is Human: Building a Safer Health System. Washington, DC: National Academy Press, 2000. https://doi.org/10.1016/s1051-0443(01)70072-3

2. Reason J. Human error: Models and management. BMJ 2001;320(7237):768-770. https://doi.org/10.1136/ bmj. 320.7237 .768

3. Chang A, Schyve PM, Croteau RJ, O'Leary DS, Loeb JM. The JCAHO patient safety event taxonomy: A standardized terminology and classification schema for near misses and adverse events. Int J Qual Health Care 2005;17(2):95-105. https://doi.org/10.1093/intghc/mzi021
4. Clarke DL, Gouveia J, Thomson SR, Muckart DJ. Applying modern error theory to the problem of missed injuries in trauma. World J Surg 2008:32(6): 1176-1182. https://doi.org/10.1007/s00268-008-9543-7

5. Gruen RL, Jurkovich GJ, McIntyre LK, Foy HM, Maier RV. Patterns of errors contributing to trauma Gruen RL, Jurkovich GJ, McIntyre LK, Foy HM, Maier RV. Patterns of errors contributing to trauma
mortality: Lessons learned from 2,594 deaths. Ann Surg 2006;244(3):371-380. https://doi.org/10.1016/ s0090-3671(08)70026-0

6. Teixeira PG, Inaba K, Hadjizacharia P, et al. Preventable or potentially preventable mortality at a mature trauma center. J Trauma 2007;63(6):1338-1346; discussion 1346-1347. https://doi.org/10.1097/ ta.0b013e31815078ae

7. Laing G, Bruce J, Skinner D, et al. Using a hybrid electronic medical record system for the surveillance of adverse surgical events and human error in a developing world surgical service. World J Surg 2015;39(1):70-79. https://doi.org/10.1007/s00268-014-2766-x

8. Dindo D, Demartines N, Clavien PA. Classification of surgical complications: A new proposal with evaluation in a cohort of 6336 patients and results of a survey. Ann Surg 2004;240(2):205-213. https:// doi.org/10.1097/01.sla.0000133083.54934.ae

9. Brady AP. Error and discrepancy in radiology: Inevitable or avoidable? Insights Imaging 2017;8(1):171Brady AP. Error and discrepancy in radiology:

10. Bruno MA, Walker EA, Abujudeh HH. Understanding and confronting our mistakes: The Bruno MA, Walker EA, Abujudeh HH. Understanding and confronting our mistakes: The
epidemiology of error in radiology and strategies for error reduction. Radiographics 2015;35(6):16681676. https://doi.org/10.1148/rg.201515002

11. Issa G, Taslakian B, Itani M, et al. The discrepancy rate between preliminary and official reports of emergency radiology studies: A performance indicator and quality improvement method. Acta Radiol 2015;56(5):598-604. https://doi.org/10.1177/0284185114532922

12. Aldous CM, Searle R, Clarke DL. An educational programme for error awareness in acute trauma for junior doctors. Afr J Health Prof Educ 2014;6(2):161-164. https://doi.org/10.7196/ajhpe.350

13. Clarke DL Furlong $\mathrm{H}$, Laing GL Aldous $C$. Thomson SR Using a structured morbidity and mortality meeting to understand the contribution of human error to adverse surgical events in a South African meeting to understand the contribution of human error to adverse surgical events
regional hospital. S Afr J Surg 2013;51(4):122-126. https:// doi.org/10.7196/sajs.1537

14. Bashir AA, Kong V, Skinner D, et al. Contrast-induced nephropathy following CT scan for trauma is nashir AA, Kong V, Skinner D, et al. Contrast-induced nephropathy following CT scan for trauma is
not rare and is associated with increased mortality in South African trauma patients. Eur I Trauma Emerg Surg (2018). https://doi.org/10.1007/s00068-018-1008-6

15. Treadwell JR, Lucas S, Tsou AY. Surgical checklists: A systematic review of impacts and implementation. BMJ Qual Saf 2013;23(4):299-318. https://doi.org/10.1136/bmjqs-2012-001797

Accepted 5 February 2019. 